\title{
Research on the Dynamic Scheduling Method of Logistics Park Based on Queuing Theory
}

\author{
Xuejian Zhao', Yuying Du, a, Zhe Sun ${ }^{1}$, Chun Ying ${ }^{2}$, Shuhua Tan², \\ Zhixin Sun ${ }^{1, b}$
}

${ }^{1}$ Nanjing University of Posts and Telecommunications, Nanjing 210003, China;

2the YuanTong Express, Shanghai 201705, China.

a347897805@qq.com, bsunzx@njupt.edu.cn

\begin{abstract}
Queuing theory is a mathematical theory and method for studying the random dispersion phenomenon and the working process of a random service system, and is mainly used to solve the queuing or congestion phenomenon. This article systematically studies the logistics dispatch at the present stage. Based on this, this paper proposes a dynamic vehicle scheduling method based on queuing theory. The results show that this method can effectively solve the problem of vehicle congestion in logistics parks.
\end{abstract}

Keywords: Logistics Park; Dynamic scheduling; Queuing theory.

\section{Introduction}

With the rapid development of electronic commerce and other industries, logistics companies have more business of shipping and unloading goods, which makes a large number of logistics vehicles in and out of the factory area, but these vehicles have no effective information in the plant, and they stop at random. It often leads to a series of problems, such as traffic congestion in the factory area, low unloading efficiency, late shipment and so on. And these problems also make the enterprise work inefficiently. Traditional vehicle scheduling is difficult to meet the requirements of real time, high efficiency and low cost in the current e-commerce environment. At present, the main problem for the vehicle scheduling in the logistics company park is how to dynamically schedule the logistics vehicles in the park and optimize the scheduling of each vehicle according to the specific conditions so as to improve the loading and unloading efficiency of the logistics vehicle.

There are many methods proposed to optimize the dynamic scheduling of logistics vehicles. At present, the improved genetic algorithm proposed in literature [1-2] can solve the vehicle scheduling problem well and maximize the profit. Particle swarm optimization (PSO) algorithm is proposed in literature [3] and [6] to solve vehicle parallel scheduling problem, which is effective and feasible, especially suitable for large-scale factories. Literature [4] proposed, an improved method based on ant colony algorithm was proposed. Based on the grid method, the vehicle workspace is modeled, and the foraging behavior of ant colony is simulated, and applied to vehicle path planning. Some new strategies are proposed to avoid boundary problem, scratch obstacle problem and deadlock problem. The simulation results show that the improved method can improve the efficiency and accuracy of path planning, and can quickly find the optimal path, and it is effective and feasible. In literature [5], a dynamic vehicle scheduling method based on hierarchical task network (HTN) was proposed. This method designs two kinds of dynamic vehicle scheduling strategies according to the principle of actual dispatching, which has good adaptability to solve dynamic vehicle scheduling problems, and the handling of agility abnormal services is helpful to improve the quality of bus service. Literature [7] proposed a comprehensive model of patient and staff satisfaction, that is, the effective satisfaction model with queuing theory. The method uses a waiting (waiting time) model to estimate patient satisfaction levels. The satisfaction of the staff is estimated based on the model of the patient's service time (service time). The patients' satisfaction, waiting time, the staff satisfaction and the service time, which provide experience and efficiency of nursing service, and in the design and management of health services to take a more comprehensive approach. Different techniques are used to solve the dynamic vehicle scheduling problem in the above literature, but the algorithms mentioned above are 
prone to fall into local optimum, and may appear premature convergence and so on. After analyzing and comparing genetic algorithm, particle swarm optimization algorithm and ant colony algorithm are not suitable for small scale vehicle scheduling, so these algorithms are not suitable for the vehicle scheduling problem in Logistics Park.

Therefore, a dynamic vehicle scheduling method based on queuing theory for logistics parks is used to solve the dynamic vehicle scheduling problem. The method can solve the problem of realtime scheduling of logistics vehicles when the logistics vehicles are too dense in the logistics company park. It can effectively improve the congestion of logistics vehicles in the logistics park and improve the efficiency of vehicle scheduling in the park.

\section{Method of Vehicle Scheduling Based on Queuing Theory}

\subsection{The Basic Indexes of the Queuing Systems.}

Queuing Theory, also known as stochastic service system theory, is a discipline developed to solve some problems in life. If categorized according to various possible conditions of the characteristics of the three components of the queuing system, the queuing system can be divided into an infinite variety of types. Therefore, only the main features can be classified. The classification is usually based on the time interval distribution of successive customers arriving at the system, the distribution of service hours and the number of service desks. The commonly used classification method in modern times is the classification method proposed by the British mathematician D.G. Kendall, which uses the Kendall symbol X/Y/Z for classification.

$\mathrm{X}$ fills in the distribution of successive arrival intervals;

Fill in Y service time distribution;

$\mathrm{Z}$ fill out the number of service desks.

Various distribution symbols are: M-negative exponential distribution; D-deterministic type; GIgeneral independent distribution; G-general random distribution. For example, $M / M / 1$ represents a model where the customer's successive arrival time is a negative exponential distribution, service time is a negative exponential distribution, and a single service desk. $\mathrm{D} / \mathrm{M} / \mathrm{C}$ denotes a model in which the customer arrives at a determined interval, the service time is a negative exponential distribution, and $\mathrm{C}$ service desks. As for some other features, such as unlimited sources or limited sources for customers, additional explanations can be provided on the basis of basic classification.

This paper proposes a dynamic scheduling method for logistics vehicles based on queuing theory. Based on the relevant information sent by each logistics vehicle to the dispatch center, the exact location of the logistics vehicle is determined. According to the specific location of the logistics vehicle, a multi-service window waiting queue model is adopted. In order to facilitate the study of the queuing model, the queuing model was hypothesized according to the three elements of the queuing model:

(1) The service order of loading and unloading sites is based on "first come first served".

(2) A logistics vehicle is a vehicle that has already reached the park. The arrival of the logistics vehicle is random, individual, and independent. The time interval between each other obeys the exponential distribution. The number of arriving customers within a certain period obeys the Poisson distribution.

(3) There are multiple service desks in the system. Each service desk can only serve one logistics vehicle at a time, and it is assumed that the service capabilities of each service desk are the same. When the logistics vehicle arrives, if there is a free service desk, service is provided, and if the service desk is busy, the shortest queue is selected for waiting.

(4) The service time and the arrival time of the logistics vehicle are independent of each other, and the logistics vehicles of each service desk in the system are arranged in a team.

Since the logistics vehicles are randomly arriving at the logistics park and obey the Poisson distribution, the probability of reaching $n$ logistics vehicles within time $t$ is 


$$
P_{n}(t)=\frac{e^{-\lambda_{\mathrm{t} \cdot} \cdot \lambda_{\mathrm{t})^{n}}}}{n !} \quad(\mathrm{n}=1,2,3 \ldots \mathrm{N})
$$

When the logistics vehicle arrives and all the loading and unloading sites are occupied, the logistics vehicles need to wait in line, so the queuing model is waiting. For loading and unloading services for logistics vehicles, multiple loading and unloading sites are arranged in parallel, service time is random, and random service time $\mathrm{v}$ is subject to negative exponential distribution. Its distribution function is

$$
\mathrm{P}(\mathrm{v} \leq \mathrm{t})=1-\mathrm{e}^{-\mu \mathrm{t}}(t \geq 0)
$$

Where $\mu$ is the average service rate and $1 / \mu$ is the average service time.

\subsection{The M/M/1 Model for Single-Server Queues.}

This is the simplest queuing system to analyze. The arrival and service time here is a negative exponential distribution (Poisson process). The system contains only one server. This queuing system can be applied to a variety of problems, because any system with a large number of independent customers can be approximately a Poisson process: $P_{n}=P\{N=n\}, n=0,1,2 \ldots$, is the probability distribution of queue length, because the queue system is in equilibrium,

$$
\rho=\frac{\lambda}{\mu}<1, P_{n}=(1-\rho) \rho^{n}, n=1,2,3 \ldots
$$

The mean queue length is

$$
\mathrm{L}=\sum_{n=0}^{\infty} n P_{n}=\sum_{n=0}^{\infty} n(1-\rho) \rho^{n}=\frac{\rho}{1-\rho}=\frac{\lambda}{\mu-\lambda}
$$

The mean queuing length is

$$
L_{q}=\sum_{n=0}^{\infty}(n-1) P_{n}=\frac{\rho^{2}}{1-\rho}=\frac{\lambda^{2}}{\mu(\mu-\lambda)}
$$

The mean staying time is

$$
\mathrm{W}=\mathrm{E}(\mathrm{T})=\frac{1}{\mu-\lambda}
$$

The mean waiting time is

$$
W_{q}=W-\frac{1}{\mu}=\frac{\lambda}{\mu(\mu-\lambda)}
$$

\subsection{The M/M/N Model for Multi-Server Queues.}

Suppose the logistics vehicle arrives according to the Poisson distribution of parameters $\lambda(\lambda>0)$. Because the service time required for each logistics vehicle is independent, it is assumed to obey the negative exponential distribution of the same parameter $\mu(\mu>0)$. Suppose there are $n$ logistics vehicles arriving, and each regional branch loading and unloading site has $\mathrm{m}$ service desks.

Let $\rho=\frac{\lambda}{\mu}, \rho_{m}=\frac{\rho}{m}=\frac{\lambda}{m \mu}, \rho$ is a yardstick for measuring the ability of loading and unloading sites to undertake loading and unloading tasks and meet their needs. The indicators in the steady state are: let $P_{n}=\lim _{t \rightarrow \infty} P\{N(t)=n\}, n=0,1,2 \ldots$, when $P_{m}<1, \exists\left\{P_{n}, n \geq 0\right\}$, independent of initial conditions. And there are: 


$$
P_{n}=\left\{\begin{array}{c}
\frac{1}{n !} \rho^{n} P_{0}, 1 \leq n \leq m-1 \\
\frac{1}{m ! m^{n-m}} \rho^{n} P_{0}, n \geq m
\end{array}\right.
$$

The probability of no vehicle in the system is

$$
P_{0}=\left[\sum_{n=0}^{m-1} \frac{\rho^{n}}{n !}+\frac{\rho^{m}}{m !\left(1-\frac{\rho}{m}\right)}\right]^{-1}
$$

Whenn $\geq \mathrm{m}$, the next logistics vehicle needs to wait, that is

$$
\mathrm{C}(\mathrm{m}, \rho)=\sum_{n=m}^{\infty} P_{n}=\frac{\rho^{m}}{m !\left(1-\rho_{m}\right)} P_{0}
$$

The mean queuing length is

$$
L_{q}=\frac{\mathrm{C}(\mathrm{m}, \rho) \rho_{m}}{1-\rho_{m}}
$$

The mean queue length is

$$
\mathrm{L}=L_{q}+\rho
$$

The mean waiting time is

$$
W_{q}=\frac{L_{q}}{\lambda}
$$

The mean staying time is

$$
\mathrm{W}=\frac{\mathrm{L}}{\lambda}
$$

\section{Instance Verification}

We assume that a logistics park dynamic scheduling method based on queuing theory is used in a logistics park. There are several regional branch loading and unloading sites in the logistics park, and each regional branch has a corresponding loading and unloading point, such as Shanghai loading and unloading point, Suzhou. Loading and unloading points, Nanjing loading and unloading points, Inner Mongolia loading and unloading points, etc. When the logistics vehicle enters the logistics park, there are special staff and equipment to collect and confirm the relevant information of the vehicle, including the license plate number of the vehicle, the relevant information of the driver, and the number of goods in the vehicle. These goods are which destination site to send to. After the relevant staff has summarized and confirmed the information, they can inform the driver that the logistics vehicle needs to go to its corresponding regional branch loading and unloading point for cargo handling. Based on the three elements of queuing theory, we conduct the following analysis of logistics parks:

(1) Input process: Logistics vehicles arrive at the park in turn, and the arrival of the logistics vehicles is random, individual and independent. The time interval between them obeys the exponential distribution, and the logistics vehicles arriving within a certain period obey the Poisson distribution. 
(2) Service process: There are several regional branch loading and unloading points in the logistics park. Each regional branch can have multiple service desks. Each service desk can only serve one logistics vehicle at a time, and assumes the service capabilities of each service desk. Are the same. When the logistics vehicle arrives, if there is a free service desk, service is provided, and if the service desk is busy, the shortest queue is selected for waiting.

(3) Service rules: The service order of loading and unloading sites is based on the first-come, firstserved rules.

According to the above three factors, we can establish a queuing model for multi-service window waiting. We then study how many service desks are set up in each regional division according to the actual situation to effectively reduce the waiting time of logistics vehicles.

First of all, according to the actual situation, we assume that the regional divisions with large cargo volume such as Shanghai and Nanjing are A loading and unloading points, similar to the regional loading divisions with smaller cargo volume such as Inner Mongolia.

Assume that 4 logistics vehicles need to be loaded and unloaded at the A loading and unloading point every hour. The loading and unloading capacity of each service station at the A loading and unloading point is the same, and the loading and unloading work of one logistics vehicle is completed in an average of 2.5 hours. Obeying the negative exponential distribution, there are now one service desk, two service desks, and three service desks according to the loading and unloading point to calculate the corresponding queuing indicators.

When the A loading and unloading station has a service station, the $\mathrm{M} / \mathrm{M} / 1$ queuing theory model is satisfied, and $\mathrm{m}=1, \lambda=4, \mu=2.5, \rho=\frac{\lambda}{\mu}=\frac{8}{5}$. At this time

$$
\begin{gathered}
\mathrm{L}=\sum_{n=0}^{\infty} n P_{n}=\sum_{n=0}^{\infty} n(1-\rho) \rho^{n}=2.889 ; L_{q}=\sum_{n=0}^{\infty}(n-1) P_{n}=4.667 ; \mathrm{W}=\mathrm{E}(\mathrm{T})=0.889 \\
W_{q}=W-\frac{1}{\mu}=1.267
\end{gathered}
$$

When the A loading and unloading station has two service stations, the $M / M / 2$ queuing theory model is satisfied, and $\mathrm{m}=2, \lambda=4, \mu=2.5, \rho=\frac{\lambda}{\mu}=\frac{8}{5}$. The idle probability at this time is

$$
P_{0}=\left[\sum_{n=0}^{m-1} \frac{\rho^{n}}{n !}+\frac{\rho^{m}}{m !\left(1-\frac{\rho}{m}\right)}\right]^{-1}=0.111
$$

Then the probability that the next logistics vehicle needs to wait when it arrives is

$$
\begin{gathered}
\mathrm{C}(\mathrm{m}, \rho)=\sum_{n=m}^{\infty} P_{n}=\frac{\rho^{m}}{m !\left(1-\rho_{m}\right)} P_{0}=0.711 ; L_{q}=\frac{\mathrm{C}(\mathrm{m}, \rho) \rho_{m}}{1-\rho_{m}}=2.844 ; L=L_{q}+\rho=4.444 ; \\
W_{q}=\frac{L_{q}}{\lambda}=0.711 ; W=\frac{\mathrm{L}}{\lambda}=1.111
\end{gathered}
$$

When the A loading and unloading station has three service stations, the $M / M / 3$ queuing theory model is satisfied, and $\mathrm{m}=3, \lambda=4, \mu=2.5, \rho=\frac{\lambda}{\mu}=\frac{8}{5}$. The idle probability at this time is

$$
P_{0}=\left[\sum_{n=0}^{m-1} \frac{\rho^{n}}{n !}+\frac{\rho^{m}}{m !\left(1-\frac{\rho}{m}\right)}\right]^{-1}=0.187
$$

Then the probability that the next logistics vehicle needs to wait when it arrives is

$$
\mathrm{C}(\mathrm{m}, \rho)=\sum_{n=m}^{\infty} P_{n}=\frac{\rho^{m}}{m !\left(1-\rho_{m}\right)} P_{0}=0.274 ; L_{q}=\frac{\mathrm{C}(\mathrm{m}, \rho) \rho_{m}}{1-\rho_{m}}=0.313 ; L=L_{q}+\rho=1.913
$$




$$
W_{q}=\frac{L_{q}}{\lambda}=0.078 ; \mathrm{W}=\frac{\mathrm{L}}{\lambda}=0.478
$$

Table 1. A loading and unloading site setting multiple service desk comparison

\begin{tabular}{|c|c|c|c|}
\hline \hline Idle probability & $\mathrm{M} / \mathrm{M} / 1$ & $\mathrm{M} / \mathrm{M} / 2$ & $\mathrm{M} / \mathrm{M} / 3$ \\
\hline & & 0.111 & 0.187 \\
\hline Waiting probability & & 0.711 & 0.274 \\
\hline The mean queuing length & 2.889 & 2.844 & 0.313 \\
\hline The mean queue length & 4.667 & 4.444 & 1.913 \\
\hline The mean waiting time & 0.889 & 0.711 & 0.078 \\
\hline The mean staying time & 1.267 & 1.111 & 0.478 \\
\hline
\end{tabular}

As can be seen from the above table, the waiting time and stay time due to the establishment of 1 and 2 service desks The difference is very large. Since the waiting time for setting up two or three service desks is not much different from the staying time, it is recommended to set up two service desks in a regional loading and unloading point similar to the A-loading station.

It is assumed that one logistics vehicle needs to be loaded and unloaded at the B loading and unloading point every three hours. The loading and unloading capacity of each service station of the B loading and unloading point is the same, and the loading and unloading work of one logistics vehicle is completed in an average of 2.5 hours. Obeying the negative exponential distribution, there is now one service desk and two service desks according to the loading and unloading point to calculate the corresponding queuing indicators.

When the B loading and unloading station has a service station, the $\mathrm{M} / \mathrm{M} / 1$ queuing theory model is satisfied, where $\mathrm{m}=1, \lambda=\frac{1}{3}, \mu=2.5, \rho=\frac{\lambda}{\mu}=\frac{2}{15}$. At this time:

$$
\begin{gathered}
\mathrm{L}=\sum_{n=0}^{\infty} n P_{n}=\sum_{n=0}^{\infty} n(1-\rho) \rho^{n}=0.154 ; L_{q}=\sum_{n=0}^{\infty}(n-1) P_{n}=0.021 ; \\
W_{q}=W-\frac{1}{\mu}=0.062 ; \mathrm{W}=\mathrm{E}(\mathrm{T})=0.462
\end{gathered}
$$

When the $B$ loading and unloading station has two service stations, the $M / M / 2$ queuing theory model is satisfied, and $\mathrm{m}=2, \lambda=\frac{1}{3}, \mu=2.5, \rho=\frac{\lambda}{\mu}=\frac{2}{15}$. The idle probability at this time is

$$
P_{0}=\left[\sum_{n=0}^{m-1} \frac{\rho^{n}}{n !}+\frac{\rho^{m}}{m !\left(1-\frac{\rho}{m}\right)}\right]^{-1}=0.929
$$

Then the probability that the next logistics vehicle needs to wait when it arrives is

$$
\begin{gathered}
\mathrm{C}(\mathrm{m}, \rho)=\sum_{n=m}^{\infty} P_{n}=\frac{\rho^{m}}{m !\left(1-\rho_{m}\right)} P_{0}=0.009 ; L_{q}=\frac{\mathrm{C}(\mathrm{m}, \rho) \rho_{m}}{1-\rho_{m}}=0.0006 ; L=L_{q}+\rho=0.134 \\
W_{q}=\frac{L_{q}}{\lambda}=0.002 ; W=\frac{\mathrm{L}}{\lambda}=0.402
\end{gathered}
$$

Table 2. B loading and unloading site setting multiple service desk comparison

\begin{tabular}{|c|c|c|}
\hline \hline & $\mathrm{M} / \mathrm{M} / 1$ & $\mathrm{M} / \mathrm{M} / 2$ \\
\hline Idle probability & & 0.929 \\
\hline Waiting probability & & 0.009 \\
\hline The mean queuing length & 0.154 & 0.0006 \\
\hline The mean queue length & 0.021 & 0.134 \\
\hline The mean waiting time & 0.062 & 0.002 \\
\hline The mean staying time & 0.462 & 0.402 \\
\hline
\end{tabular}


As can be seen from the above table, the waiting time and stay time of setting up one and two service desks are not much different, considering the cost of building a logistics park, we do not need to set up multiple services for each regional branch loading and unloading point. Taiwan, we need to achieve the best way to reduce vehicle waiting time and logistics park construction costs at the same time, so in a regional loading and unloading point similar to the B loading and unloading site, it is recommended to set up only one service desk.

Considering the cost of building a logistics park, we don't need to set up multiple loading and unloading operations in each regional branch. In the regions with a large number of express shipments, a number of loading and unloading sites are set up, and the regional branches with less express shipments are only set up. A loading and unloading site. The establishment of one more site per regional branch can significantly reduce the waiting time of logistics vehicles. Considering the construction cost of the logistics park, we can set up 2-3 loading and unloading stations in the regional branches with large express shipments, while only a few loadings and unloading stations are set up in fewer regional branches, so that the queue rate of logistics vehicles will be greatly reduced. It can also greatly improve the efficiency of vehicle scheduling in the logistics park.

\section{Summary}

This paper aims at the vehicle congestion in the park caused by the random parking of vehicles in the logistics park at this stage. After analysis, taking into account the dynamic scheduling problem of the vehicle, a dynamic scheduling method based on queuing theory in the logistics park is proposed. Using the queuing model to obtain the optimal vehicle scheduling scheme can solve the problem of vehicle congestion in the logistics park. And the operation is simple and easy to implement. Of course, this method still has some deficiencies, and further research is needed in the future work.

\section{Acknowledgments}

This work is supported by the National Engineering Laboratory for Logistics Information Technology of Yuan Tong Express, the Natural Science Foundation of Jiangsu Province No. BK20160913 and No.BK20140883, the High Level Teacher Research Foundation of Nanjing University of Posts and Telecommunications No.NY2016021, The Chinese Natural Science Incubation Foundation of Nanjing University of Posts and Telecommunications No.NY217055, Jiangsu Postdoctoral Foundation No.1701016A, Natural Science Foundation of China No.61602259,No.61373135,No.61672299.

\section{References}

[1]. Zuo X, Chen C, Tan W, et al. Vehicle Scheduling of an Urban Bus Line via an Improved Multiobjective Genetic Algorithm[J]. IEEE Transactions on Intelligent Transportation Systems, 2015.

[2]. Prata B D A. A hybrid Genetic Algorithm for the vehicle and crew scheduling in mass transit systems[J]. IEEE Latin America Transactions, 2015, 13(9):3020-3025.

[3]. Tian G, Ren Y, Zhou M C. Dual-Objective Scheduling of Rescue Vehicles to Distinguish Forest Fires via Differential Evolution and Particle Swarm Optimization Combined Algorithm[M]. IEEE Press, 2016.

[4]. Zhang Y, Cao Y, Han Z. Path planning of vehicle based on improved ant colony algorithm[C]// Proceedings of International Conference on Modelling, Identification \& Control. IEEE, 2012.

[5]. Shen Y, Zeng Z, Wu Z. Dynamic vehicle scheduling based on HTN[C]// Chinese Control Conference (international. 2017:3066-3071. 
[6]. Wang X, Jiang M, Chen S, et al. A Hybrid Particle Swarm Optimization for Parallel Machine Scheduling with Shared and Multi-mode Resources[C]// International Symposium on Computational Intelligence and Design. IEEE, 2017:429-433.

[7]. Komashie A, Mousavi A, Clarkson P J, et al. An Integrated Model of Patient and Staff Satisfaction Using Queuing Theory[J]. IEEE Journal of Translational Engineering in Health \& Medicine, 2015. 